Provided for non-commercial research and education use. Not for reproduction, distribution or commercial use.

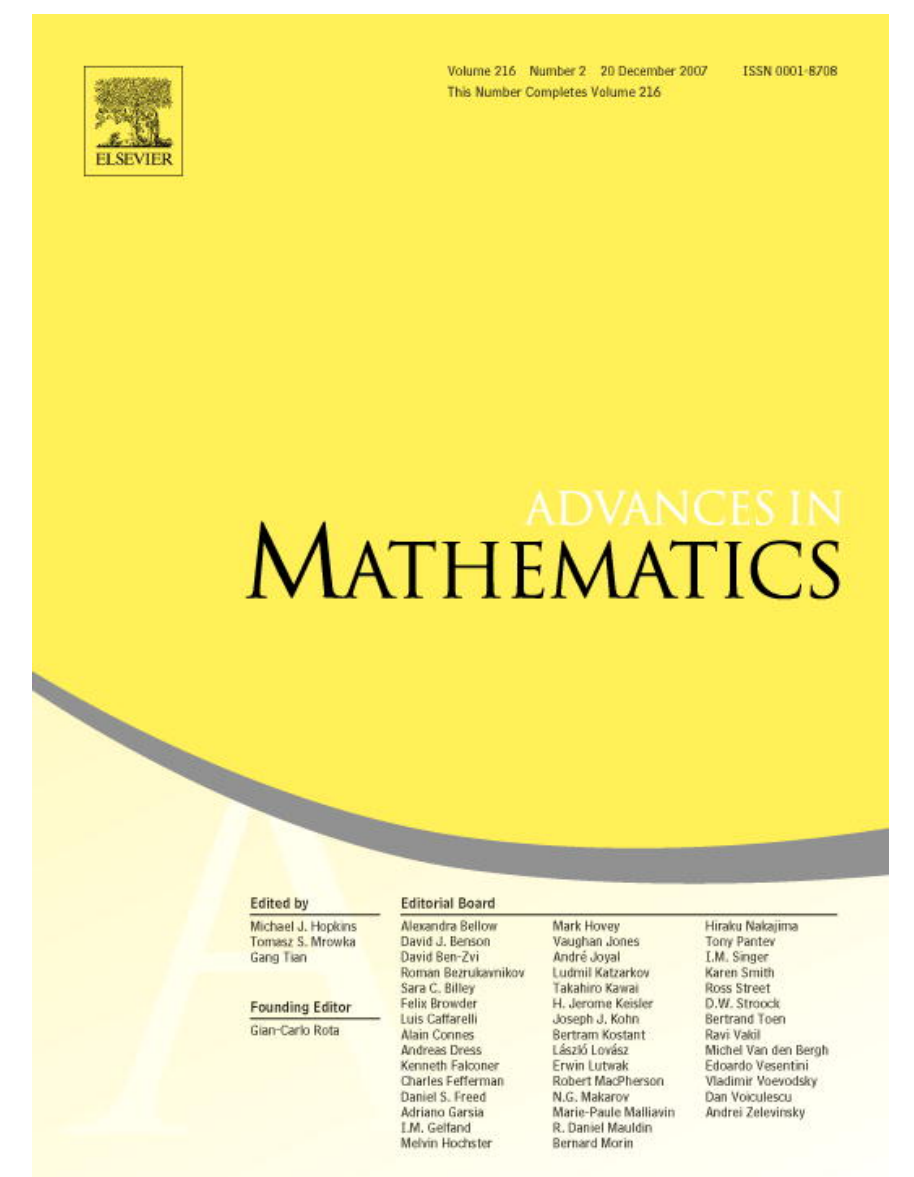

This article was published in an Elsevier journal. The attached copy

is furnished to the author for non-commercial research and education use, including for instruction at the author's institution, sharing with colleagues and providing to institution administration.

Other uses, including reproduction and distribution, or selling or licensing copies, or posting to personal, institutional or third party websites are prohibited.

In most cases authors are permitted to post their version of the article (e.g. in Word or Tex form) to their personal website or institutional repository. Authors requiring further information regarding Elsevier's archiving and manuscript policies are encouraged to visit:

http://www.elsevier.com/copyright 


\title{
Sixth order mock theta functions
}

\author{
Bruce C. Berndt ${ }^{\mathrm{a}, *, 1}$, Song Heng Chan ${ }^{\mathrm{b}, 2}$ \\ ${ }^{a}$ Department of Mathematics, University of Illinois, 1409 West Green Street, Urbana, IL 61801, USA \\ ${ }^{\mathrm{b}}$ Department of Mathematics, National University of Singapore, 2 Science Drive 2, \\ Singapore 117543, Republic of Singapore
}

Received 18 December 2006; accepted 5 June 2007

Available online 13 June 2007

Communicated by Michael J. Hopkins

\begin{abstract}
Two new mock theta functions of the sixth order are defined. The main theorem in this paper (Theorem 1.1) provides four transformation formulas relating the new mock theta functions with Ramanujan's mock theta functions of the sixth order. Two further representations of the new mock theta functions are established. Lastly, a hitherto unproved entry from Ramanujan's lost notebook related to sixth order mock theta functions is proved.
\end{abstract}

(c) 2007 Elsevier Inc. All rights reserved.

MSC: primary 33D15; secondary $11 \mathrm{~F} 27$

Keywords: Mock theta functions; Ramanujan's lost notebook; Theta functions; $q$-series; Bailey pairs; Constant term method

\section{Introduction}

In Ramanujan's last letter to G.H. Hardy [12, pp. xxxi-xxxii, 354-355], [5, pp. 220-223], written on January 12, 1920, Ramanujan introduced mock theta functions and gave examples of mock theta functions of the third, fifth, and seventh orders. Ramanujan did not rigorously define a mock theta function, nor did he define the order of a mock theta function. Although several mathematicians have since given their definitions of the order of a mock theta function,

\footnotetext{
* Corresponding author.

E-mail addresses: berndt@math.uiuc.edu (B.C. Berndt), chansh@ntu.edu.sg (S.H. Chan).

1 Research is partially supported by grant MDA904-00-1-0015 from the National Security Agency.

2 Present address: Division of Mathematical Sciences, School of Physical and Mathematical Sciences, Nanyang Technological University, Block 5, Level 3, Singapore 637616, Republic of Singapore.
} 
up until recent times there was no widely accepted definition of a mock theta function or of its order. However, the recent work of K. Bringmann and K. Ono [6-8] demonstrating that mock theta functions are the holomorphic parts of Maass wave forms has clarified the concept of order. When Ramanujan's lost notebook was found by G.E. Andrews in 1976, it was seen to contain identities for mock theta functions of the sixth and tenth orders. We do not repeat Ramanujan's definition of a mock theta function here, but it is widely accepted that the only other mock theta functions that have been discovered besides those of Ramanujan are third order mock theta functions defined by G.N. Watson [14] and Andrews [1].

The first goal of this paper is to define two new sixth order mock theta functions, which have their origins in Ramanujan's mock theta functions of the sixth order. In Theorem 1.1 below, we offer four identities, which are similar in spirit to identities for sixth order mock theta functions stated by Ramanujan in his lost notebook [13] and proved by Andrews and D. Hickerson [3]. Most of this paper is devoted to proving these four identities. In fact, R.J. McIntosh [11] independently discovered these two mock theta functions and derived transformation formulas for them. There are no theorems in [11] in common with those proved in our paper.

Throughout this article, we assume that $|q|<1$ and use the notation

$$
\begin{aligned}
(x)_{0} & :=(x ; q)_{0}:=1, \\
(x)_{n} & :=(x ; q)_{n}:=\prod_{k=0}^{n-1}\left(1-x q^{k}\right), \\
\left(x_{1}, \ldots, x_{m}\right)_{n} & :=\left(x_{1}, \ldots, x_{m} ; q\right)_{n}:=\left(x_{1} ; q\right)_{n} \cdots\left(x_{m} ; q\right)_{n}, \\
{\left[x_{1}, \ldots, x_{m}\right]_{n} } & :=\left[x_{1}, \ldots, x_{m} ; q\right]_{n}:=\left(x_{1}, q / x_{1}, \ldots, x_{m}, q / x_{m} ; q\right)_{n} .
\end{aligned}
$$

Recall the six primary sixth order mock theta functions defined in [3], namely,

$$
\begin{aligned}
\phi(q) & :=\sum_{n=0}^{\infty} \frac{(-1)^{n} q^{n^{2}}\left(q ; q^{2}\right)_{n}}{(-q ; q)_{2 n}} \\
\psi(q) & :=\sum_{n=1}^{\infty} \frac{(-1)^{n-1} q^{n^{2}}\left(q ; q^{2}\right)_{n-1}}{(-q ; q)_{2 n-1}} \\
\rho(q) & :=\sum_{n=0}^{\infty} \frac{q^{n(n+1) / 2}(-q ; q)_{n}}{\left(q ; q^{2}\right)_{n+1}} \\
\sigma(q) & :=\sum_{n=0}^{\infty} \frac{q^{(n+2)(n+1) / 2}(-q ; q)_{n}}{\left(q ; q^{2}\right)_{n+1}} \\
\lambda(q) & :=\sum_{n=0}^{\infty} \frac{(-1)^{n} q^{n}\left(q ; q^{2}\right)_{n}}{(-q ; q)_{n}}
\end{aligned}
$$

and

$$
\mu(q):=\sum_{n=0}^{\infty} \frac{(-1)^{n}\left(q ; q^{2}\right)_{n}}{(-q ; q)_{n}}
$$


Note that the series defining $\mu(q)$ does not converge; consult [3] for a proper interpretation of this definition of $\mu(q)$. If we sum the summands of $\rho(q)$ and $\sigma(q)$ over the negative integers, we obtain $\lambda(q) / 2$ and $\mu(q) / 2$, respectively. However, an examination of the summands of $\phi(q)$ and $\psi(q)$ over the negative and non-positive integers, respectively, leads to the discovery of two new functions, which we define as

$$
\begin{aligned}
& \phi_{-}(q):=\sum_{n=1}^{\infty} \frac{q^{n}(-q)_{2 n-1}}{\left(q ; q^{2}\right)_{n}}, \\
& \psi_{-}(q):=\sum_{n=1}^{\infty} \frac{q^{n}(-q)_{2 n-2}}{\left(q ; q^{2}\right)_{n}} .
\end{aligned}
$$

Unfortunately, this method of constructing new functions does not yield any new functions when applied to Ramanujan's fifth, seventh, and tenth order mock theta functions. As remarked above, these two functions, $\phi_{-}(q)$ and $\psi_{-}(q)$, were independently discovered by McIntosh [11].

In Section 2, we prove the following analogs of $(0.18)_{R}-(0.21)_{R}$ in [3].

Theorem 1.1. In the notation above,

$$
\begin{aligned}
\rho(q)-2 q^{-1} \psi_{-}\left(q^{2}\right) & =\left(-q^{2} ; q^{2}\right)_{\infty}^{3}\left(q^{6}, q^{6}, q^{12} ; q^{12}\right)_{\infty} \\
\sigma(q)-\phi_{-}\left(q^{2}\right) & =q\left(-q^{2} ; q^{2}\right)_{\infty}^{2}\left(-q^{6},-q^{6}, q^{6} ; q^{6}\right)_{\infty} \\
4 \phi_{-}\left(q^{2}\right)+2 \mu(q) & =\left(-q ; q^{2}\right)_{\infty}^{2}\left(-q^{3},-q^{3}, q^{6} ; q^{6}\right)_{\infty} \\
4 q^{-1} \psi_{-}\left(q^{2}\right)+\lambda(q) & =\left(-q ; q^{2}\right)_{\infty}^{3}\left(q^{3}, q^{9}, q^{12} ; q^{12}\right)_{\infty}
\end{aligned}
$$

As in the paper by Andrews and Hickerson [3], we employ the Bailey pair method and the constant term method to prove Theorem 1.1. We state without proofs various results needed in our proofs.

First, we need the Jacobi triple product identity [4, pp. 33-36]. For $|a b|<1$,

$$
f(a, b):=\sum_{n=-\infty}^{\infty} a^{n(n+1) / 2} b^{n(n-1) / 2}=(-a ; a b)_{\infty}(-b ; a b)_{\infty}(a b ; a b)_{\infty} .
$$

Theorem 1.2. (See [3, Theorem 1.1].) If $n$ is a positive integer, $0<|q|<1$, and neither $x$ nor $z$ is 0 or an integral power of $q$, then

$$
\frac{[x z ; q]_{\infty}\left[x^{n} ; q^{n}\right]_{\infty}(q ; q)_{\infty}^{2}}{[x, z ; q]_{\infty}\left(q^{n} ; q^{n}\right)_{\infty}^{2}}=\sum_{k=0}^{n-1} \frac{x^{k}\left[q^{k} x^{n} z ; q^{n}\right]_{\infty}}{\left[q^{k} z ; q^{n}\right]_{\infty}} .
$$

The proof of Theorem 1.2 is dependent on a corollary of Ramanujan's ${ }_{1} \psi_{1}$ summation formula [9, Eq. (3.1)], namely,

$$
\frac{[x z ; q]_{\infty}(q ; q)_{\infty}^{2}}{[x, z ; q]_{\infty}}=\sum_{n=-\infty}^{\infty} \frac{x^{n}}{1-z q^{n}}
$$


for $|q|<|x|<1$. Expanding the right side as a double series gives

$$
\begin{aligned}
\frac{[x z ; q]_{\infty}(q ; q)_{\infty}^{2}}{[x, z ; q]_{\infty}} & =\sum_{n=-\infty}^{\infty} \frac{x^{n}}{1-z q^{n}}=\sum_{n=0}^{\infty} \frac{x^{n}}{1-z q^{n}}-\sum_{n=1}^{\infty} \frac{q^{n} /\left(x^{n} z\right)}{1-q^{n} / z} \\
& =\sum_{n=0}^{\infty} \sum_{m=0}^{\infty} x^{n} z^{m} q^{m n}-\sum_{n=1}^{\infty} \sum_{m=1}^{\infty} x^{-n} z^{-m} q^{m n}
\end{aligned}
$$

provided also that $|q|<|z|<1$.

Definition 1.3. (See [3, Definition 2.0].) Two sequences $\left\{\alpha_{n}\right\}$ and $\left\{\beta_{n}\right\}, n \geqslant 0$, form a Bailey pair relative to a number $a$ if

$$
\beta_{n}=\sum_{r=0}^{n} \frac{\alpha_{r}}{(q)_{n-r}(a q)_{n+r}}
$$

for all $n \geqslant 0$.

Corollary 1.4. (See [3, Corollary 2.1].) If $\left\{\alpha_{n}\right\}$ and $\left\{\beta_{n}\right\}$ form a Bailey pair relative to a, then

$$
\sum_{n=0}^{\infty} \frac{\left(\rho_{1}\right)_{n}\left(\rho_{2}\right)_{n}\left(a q /\left(\rho_{1} \rho_{2}\right)\right)^{n} \alpha_{n}}{\left(a q / \rho_{1}\right)_{n}\left(a q / \rho_{2}\right)_{n}}=\frac{(a q)_{\infty}\left(a q /\left(\rho_{1} \rho_{2}\right)\right)_{\infty}}{\left(a q / \rho_{1}\right)_{\infty}\left(a q / \rho_{2}\right)_{\infty}} \sum_{n=0}^{\infty}\left(\rho_{1}\right)_{n}\left(\rho_{2}\right)_{n}\left(a q /\left(\rho_{1} \rho_{2}\right)\right)^{n} \beta_{n}
$$

provided both sums converge absolutely.

In [3], Andrews and Hickerson proved the following identities; their exact locations are [3, Eqs. (3.19), (4.7), (4.8), (4.19), (4.20)], respectively:

$$
\begin{aligned}
\left(z, q^{3} / z, q^{3} ; q^{3}\right)_{\infty} \psi(q)= & \frac{z(q ; q)_{\infty}\left(q^{3} ; q^{3}\right)_{\infty}\left(q, q^{5}, q^{6} ; q^{6}\right)_{\infty}\left[-z q,-z q^{2} ; q^{3}\right]_{\infty}}{\left(q^{2} ; q^{2}\right)_{\infty}\left[z ; q^{3}\right]_{\infty}} \\
& -\sum_{n=-\infty}^{\infty} \frac{(-1)^{n} z^{n+1} q^{3 n(n+1) / 2}}{1-z q^{3 n}}, \\
q\left(z, q^{6} / z, q^{6} ; q^{6}\right)_{\infty} \rho(q)=- & \frac{z\left(q^{3} ; q^{3}\right)_{\infty}^{2}\left[z q ; q^{2}\right]_{\infty}}{\left[q ; q^{2}\right]_{\infty}\left[z ; q^{3}\right]_{\infty}}+\sum_{n=-\infty}^{\infty} \frac{(-1)^{n} z^{n+1} q^{3 n(n+1)}}{1-z q^{6 n}} \\
\left(z, q^{6} / z, q^{6} ; q^{6}\right)_{\infty} \sigma(q)=- & \frac{z\left(q, q^{5}, q^{6}, q^{6} ; q^{6}\right)_{\infty}\left[q z ; q^{2}\right]_{\infty}}{\left[q ; q^{2}\right]_{\infty}\left[q z, q^{2} z ; q^{6}\right]_{\infty}} \\
& +\sum_{n=-\infty}^{\infty} \frac{(-1)^{n} z^{n+1} q^{3 n(n+1)}}{1-z q^{6 n+2}}
\end{aligned}
$$




$$
\begin{aligned}
q\left(z, q^{6} / z, q^{6} ; q^{6}\right)_{\infty} \lambda(q)= & \frac{2 z\left(q^{12} ; q^{12}\right)_{\infty}^{2}\left(q^{2} ; q^{4}\right)_{\infty}^{2}\left[-z q^{2},-z q^{4} ; q^{6}\right]_{\infty}}{\left[z ; q^{6}\right]_{\infty}} \\
& +\frac{q(q ; q)_{\infty}\left(q^{6} ; q^{6}\right)_{\infty}^{2}\left[q, z ; q^{6}\right]_{\infty}}{\left(-q,-q^{3}, q^{4} ; q^{4}\right)_{\infty}} \\
& -2 \sum_{n=-\infty}^{\infty} \frac{(-1)^{n} z^{n+1} q^{3 n(n+1)}}{1-z q^{6 n}}
\end{aligned}
$$

and

$$
\begin{aligned}
2\left(z, q^{6} / z, q^{6} ; q^{6}\right)_{\infty} \mu(q)= & \frac{2\left(q^{2} ; q^{2}\right)_{\infty}\left(q^{6} ; q^{6}\right)_{\infty}^{2}\left(q^{6} ; q^{12}\right)_{\infty}\left[-z,-z q^{2} ; q^{6}\right]_{\infty}}{\left(q^{4} ; q^{4}\right)_{\infty}\left[z q^{2} ; q^{6}\right]_{\infty}} \\
& -\frac{(q ; q)_{\infty}\left(q ; q^{2}\right)_{\infty}\left(-q,-q^{2}, q^{3} ; q^{3}\right)_{\infty}\left(z, q^{6} / z, q^{6} ; q^{6}\right)_{\infty}}{\left(-q,-q^{3}, q^{4} ; q^{4}\right)_{\infty}} \\
& -4 \sum_{n=-\infty}^{\infty} \frac{(-1)^{n} z^{n+1} q^{3 n(n+1)}}{1-z q^{6 n+2}}
\end{aligned}
$$

A similar identity for $\phi(q)$ also appears in [3, Eq. (3.20)]. We state and prove the analogous identities for $\phi_{-}(q)$ and $\psi_{-}(q)$ at the end of Section 2.

We prove a related identity on page 4 of Ramanujan's lost notebook in Section 3.

\section{Proofs of the analogous identities}

We first recall the following theorem.

Theorem 2.1. (See [3, Theorem 2.3].) Let $a, b, c$, and $q$ be complex numbers with $a \neq 1, b \neq 0$, $c \neq 0, q \neq 0$, and none of $a / b, a / c, q b$, and $q c$ of the form $q^{-k}$ with $k \geqslant 0$. For $n \geqslant 0$, define

$$
A_{n}^{\prime}(a, b, c, q)=\frac{q^{n^{2}}(b c)^{n}\left(1-a q^{2 n}\right)(a / b)_{n}(a / c)_{n}}{(1-a)(q b)_{n}(q c)_{n}} \sum_{j=0}^{n} \frac{(-1)^{j}\left(1-a q^{2 j-1}\right)(a)_{j-1}(b)_{j}(c)_{j}}{q^{j(j-1) / 2}(b c)^{j}(q)_{j}(a / b)_{j}(a / c)_{j}}
$$

and

$$
B_{n}^{\prime}(a, b, c, q)=\frac{1}{(q b)_{n}(q c)_{n}}
$$

Then the sequences $\left\{A_{n}^{\prime}(a, b, c, q)\right\}$ and $\left\{B_{n}^{\prime}(a, b, c, q)\right\}$ form a Bailey pair relative to a.

From Theorem 2.1, we know that the sequences $\left\{A_{n}^{\prime}\left(q^{4}, q, 0, q^{2}\right)\right\}$ and $\left\{B_{n}^{\prime}\left(q^{4}, q, 0, q^{2}\right)\right\}$ form a Bailey pair relative to $q^{2}$. Replacing $q$ by $q^{2}$ and replacing $\rho_{1}$ and $\rho_{2}$ by $-q^{2}$ and $-q^{3}$, respectively, in Corollary 1.4 , we find that 


$$
\begin{aligned}
\frac{(q)_{\infty}}{(-q)_{\infty}} \phi_{-}(q)= & \sum_{n=0}^{\infty}\left(1-q^{2 n+2}\right)(-1)^{n} q^{3 n^{2}+5 n+1} \sum_{j=0}^{n}\left(1+q^{2 j+1}\right) q^{-2 j^{2}-3 j} \\
= & \sum_{n=0}^{\infty}\left(1-q^{2 n+2}\right)(-1)^{n} q^{3 n^{2}+5 n+1} \sum_{j=-n-1}^{n} q^{-2 j^{2}-3 j} \\
= & \sum_{n=0}^{\infty}(-1)^{n} q^{3 n^{2}+5 n+1} \sum_{j=-n-1}^{n} q^{-2 j^{2}-3 j} \\
& +\sum_{n=1}^{\infty}(-1)^{n} q^{3 n^{2}+n-1} \sum_{j=-n}^{n-1} q^{-2 j^{2}-3 j}
\end{aligned}
$$

Similarly, the sequences $\left\{A_{n}^{\prime}\left(q^{2}, q, 0, q^{2}\right)\right\}$ and $\left\{B_{n}^{\prime}\left(q^{2}, q, 0, q^{2}\right)\right\}$ form a Bailey pair relative to $q^{2}$. Replacing $q$ by $q^{2}$ and replacing $\rho_{1}$ and $\rho_{2}$ by $-q$ and $-q^{2}$, respectively, in Corollary 1.4 , we see that

$$
\begin{aligned}
2 \frac{(q)_{\infty}}{(-q)_{\infty}} \psi_{-}(q)= & 2 \sum_{n=0}^{\infty}(-1)^{n} q^{3 n^{2}+3 n+1}\left(1+\sum_{j=1}^{n}\left(1+q^{2 j}\right) q^{-2 j^{2}-j}\right) \\
= & 2 \sum_{n=0}^{\infty}(-1)^{n} q^{3 n^{2}+3 n+1} \sum_{j=-n}^{n} q^{-2 j^{2}-j} \\
= & \sum_{n=0}^{\infty}(-1)^{n} q^{3 n^{2}+3 n+1} \sum_{j=-n}^{n} q^{-2 j^{2}-j} \\
& -\sum_{n=1}^{\infty}(-1)^{n} q^{3 n^{2}-3 n+1} \sum_{j=-n+1}^{n-1} q^{-2 j^{2}-j} .
\end{aligned}
$$

Therefore, setting $r=n+1+j$ and $s=n-j$ in (2.1), we find that

$$
\begin{aligned}
-\frac{(q)_{\infty}}{(-q)_{\infty}} \phi_{-}(q)= & \sum_{\substack{r \geqslant 0, s \geqslant 0 \\
r \neq s(\bmod 2)}}(-1)^{(r+s+1) / 2} q^{(2 r+1) s+((r+s+1) / 2)^{2}} \\
& -\sum_{\substack{r \geqslant 1, s \geqslant 1 \\
r \neq s(\bmod 2)}}(-1)^{(-r-s+1) / 2} q^{(2 r-1) s+((r+s-1) / 2)^{2}},
\end{aligned}
$$

while setting $r=n+j$ and $s=n-j$ in (2.2), we see that 


$$
\begin{aligned}
-2 \frac{(q)_{\infty}}{(-q)_{\infty}} \psi_{-}(q)= & \sum_{\substack{r \geqslant 0, s \geqslant 0 \\
r \equiv s(\bmod 2)}}(-1)^{(r+s+2) / 2} q^{(2 r+1) s+((r+s+2) / 2)^{2}} \\
& -\sum_{\substack{r \geqslant 1, s \geqslant 1 \\
r \equiv s(\bmod 2)}}(-1)^{(-r-s+2) / 2} q^{(2 r-1) s+((r+s-2) / 2)^{2}}
\end{aligned}
$$

Set

$$
B(z):=z^{2} \frac{\left(z^{2} q, q / z^{2}, q^{2}, q^{2} ; q^{2}\right)_{\infty}^{2}}{(z, q / z, q, q ; q)_{\infty}} .
$$

Then we have the following theorem.

Theorem 2.2. In the annulus $|q|<|z|<1$, the coefficients of $z^{0}$ and $z^{1}$ in $B(z)$ are $-2 \psi_{-}(q)$ and $-\phi_{-}(q)$, respectively.

Proof. By Euler's identity and the Jacobi triple product identity (1.11), we first express

$$
\begin{aligned}
\frac{(q)_{\infty}}{(-q)_{\infty}} B(z) & =z^{2} \frac{\left(z^{2} q, q / z^{2} ; q^{2}\right)_{\infty}^{2}\left(q^{2} ; q^{2}\right)_{\infty}^{3}}{(z, q / z ; q)_{\infty}} \\
& =z^{2} \frac{\left(z^{2} q, q / z^{2}, q^{2}, q^{2} ; q^{2}\right)_{\infty}}{\left(z, q^{2} / z, z q, q / z ; q^{2}\right)_{\infty}}\left(z^{2} q, q / z^{2}, q^{2} ; q^{2}\right)_{\infty} \\
& =\left(\sum_{r=0}^{\infty} \sum_{s=0}^{\infty} z^{r+s+2} q^{(2 r+1) s}-\sum_{r=1}^{\infty} \sum_{s=1}^{\infty} z^{-r-s+2} q^{(2 r-1) s}\right) \sum_{t=-\infty}^{\infty}(-1)^{t} z^{-2 t} q^{t^{2}}
\end{aligned}
$$

where in the last equality, we applied (1.13) and (1.11). Then from (2.3) and (2.4), we see that the coefficients of $z^{1}$ and $z^{0}$ in this last expression are $-\frac{(q)_{\infty}}{(-q)_{\infty}} \phi_{-}(q)$ and $-2 \frac{(q)_{\infty}}{(-q)_{\infty}} \psi_{-}(q)$, respectively.

From the Jacobi triple product identity (1.11), we deduce that

$$
(1 / z, z q, q ; q)_{\infty}=-z(z, q / z, q ; q)_{\infty}
$$

and so we easily verify that $B(z)$ satisfies the functional equations

$$
\begin{gathered}
B(z q)=-\frac{1}{z^{3}} B(z), \\
B(1 / z)=-\frac{1}{z^{3}} B(z),
\end{gathered}
$$

and it is also easy to check that the residue of $B(z)$ at $z=1$ is -1 .

Note that the Lambert series

$$
L(z):=\sum_{n=-\infty}^{\infty} \frac{(-1)^{n} q^{n(3 n+1) / 2} z^{3 n+2}}{1-q^{n} z}
$$


satisfies the same functional equations, (2.5) and (2.6). As $z \rightarrow 1$,

$$
L(z)=\frac{z^{2}}{1-z}+O(1)=-\frac{z^{2}}{z-1}+O(1),
$$

and so the residue at $z=1$ is -1 . Therefore we may conclude that the difference $V(z):=B(z)-$ $L(z)$ is analytic on the complex plane except for $z=0$, and satisfies the functional equations (2.5) and (2.6). Therefore, if

$$
V(z)=\sum_{n=-\infty}^{\infty} V_{n} z^{n}
$$

denotes the Laurent series expansion of $V(z)$ about $z=0$, the functional equation (2.5) gives us

$$
\sum_{n=-\infty}^{\infty} V_{n} z^{n}=-\sum_{n=-\infty}^{\infty} V_{n} z^{n+3} q^{n}
$$

which, upon comparing coefficients of $z^{n},-\infty<n<\infty$, yields

$$
V_{n}=-V_{n-3} q^{n-3}
$$

Therefore,

$$
\begin{aligned}
V(z)= & V_{0} \sum_{n=-\infty}^{\infty}(-1)^{n} z^{3 n} q^{3 n(n-1) / 2}+V_{1} \sum_{n=-\infty}^{\infty}(-1)^{n} z^{3 n+1} q^{n(3 n-1) / 2} \\
& +V_{2} \sum_{n=-\infty}^{\infty}(-1)^{n} z^{3 n+2} q^{n(3 n+1) / 2} \\
= & V_{0}\left(z^{3}, q^{3} / z^{3}, q^{3} ; q^{3}\right)_{\infty}+V_{1} z\left(q z^{3}, q^{2} / z^{3}, q^{3} ; q^{3}\right)_{\infty}+V_{2} z^{2}\left(q^{2} z^{3}, q / z^{3}, q^{3} ; q^{3}\right)_{\infty} \\
= & V_{0}\left(z^{3}, q^{3} / z^{3}, q^{3} ; q^{3}\right)_{\infty}+V_{1}\left\{z\left(q z^{3}, q^{2} / z^{3}, q^{3} ; q^{3}\right)_{\infty}-z^{2}\left(q^{2} z^{3}, q / z^{3}, q^{3} ; q^{3}\right)_{\infty}\right\}
\end{aligned}
$$

where in the second equality, we applied the Jacobi triple product identity (1.11), and in the third equality, we used the fact $V_{2}=-V_{1}$, which is obtained by substituting (2.8) into (2.6) and comparing the coefficients of $z$.

Recall that for $|q|<|z|<1$, Theorem 2.2 tells us that the coefficients of $z^{0}$ and $z^{1}$ in $B(z)$ are $-2 \psi_{-}(q)$ and $-\phi_{-}(q)$, respectively. Also for $|q|<|z|<1$, from (2.7), we deduce the expansion

$$
L(z)=\sum_{r=0}^{\infty} \sum_{s=0}^{\infty}(-1)^{r} q^{r(3 r+1) / 2+r s} z^{3 r+s+2}-\sum_{r=1}^{\infty} \sum_{s=1}^{\infty}(-1)^{r} q^{r(3 r-1) / 2+r s} z^{-3 r-s+2}
$$

which shows that the coefficients of $z^{0}$ and $z^{1}$ in $L(z)$ are 0 . Hence, from the definition, $V(z)=$ $B(z)-L(z)$, we conclude that $V_{0}=-2 \psi_{-}(q)$ and $V_{1}=-\phi_{-}(q)$.

Using (2.9), we obtain the following theorem. 
Theorem 2.3. We have

$$
\begin{aligned}
B(z)= & L(z)-2 \psi_{-}(q)\left(z^{3}, q^{3} / z^{3}, q^{3} ; q^{3}\right)_{\infty} \\
& -\phi_{-}(q)\left\{z\left(q z^{3}, q^{2} / z^{3}, q^{3} ; q^{3}\right)_{\infty}-z^{2}\left(q^{2} z^{3}, q / z^{3}, q^{3} ; q^{3}\right)_{\infty}\right\} .
\end{aligned}
$$

We separate $\phi_{-}(q)$ from $\psi_{-}(q)$ in (2.10). First, we apply Theorem 1.2 twice to $B(z)$ to deduce that

$$
\begin{aligned}
B(z)= & z^{2} \frac{\left(q^{6} ; q^{6}\right)_{\infty}^{4}\left[z^{3} ; q\right]_{\infty}}{(q ; q)_{\infty}^{2}\left[z^{3} ; q^{3}\right]_{\infty}}\left\{\frac{\left[q / z^{2} ; q^{2}\right]_{\infty}\left[z^{3} ; q^{6}\right]_{\infty}\left(q^{2} ; q^{2}\right)_{\infty}^{2}}{\left[z, q / z^{3} ; q^{2}\right]_{\infty}\left(q^{6} ; q^{6}\right)_{\infty}^{2}}\right\} \\
& \times\left\{\frac{\left[z^{2} q ; q^{2}\right]_{\infty}\left[q^{3} / z^{3} ; q^{6}\right]_{\infty}\left(q^{2} ; q^{2}\right)_{\infty}^{2}}{\left[q / z, z^{3} ; q^{2}\right]_{\infty}\left(q^{6} ; q^{6}\right)_{\infty}^{2}}\right\} \\
= & z^{2} \frac{\left(q^{6} ; q^{6}\right)_{\infty}^{4}\left[z^{3} ; q\right]_{\infty}}{(q ; q)_{\infty}^{2}\left[z^{3} ; q^{3}\right]_{\infty}} \sum_{k=0}^{2} \frac{z^{k}\left[q^{2 k+1} ; q^{6}\right]_{\infty}}{\left[q^{2 k+1} / z^{3} ; q^{6}\right]_{\infty}} \sum_{k=0}^{2} \frac{(q / z)^{k}\left[q^{2 k+3} ; q^{6}\right]_{\infty}}{\left[q^{2 k} z^{3} ; q^{6}\right]_{\infty}}
\end{aligned}
$$

Then letting $z=q^{2 / 3}$, we find that

$$
\begin{aligned}
B\left(q^{2 / 3}\right) & =-q^{1 / 3} \frac{\left(q^{6} ; q^{6}\right)_{\infty}^{4}\left(q^{3} ; q^{3}\right)_{\infty}}{(q ; q)_{\infty}} \sum_{k=0}^{2} \frac{q^{2 k / 3}\left[q^{2 k+1} ; q^{6}\right]_{\infty}}{\left[q^{2 k-1} ; q^{6}\right]_{\infty}} \frac{q^{2 / 3}\left[q^{7} ; q^{6}\right]_{\infty}}{\left(q^{6} ; q^{6}\right)_{\infty}^{2}} \\
& =\frac{\left(q^{6} ; q^{6}\right)_{\infty}^{2}\left(q^{3} ; q^{3}\right)_{\infty}\left[q ; q^{6}\right]_{\infty}}{(q ; q)_{\infty}} \sum_{k=0}^{2} \frac{q^{2 k / 3}\left[q^{2 k+1} ; q^{6}\right]_{\infty}}{\left[q^{2 k-1} ; q^{6}\right]_{\infty}}
\end{aligned}
$$

Next, since

$$
\frac{1}{1-q^{n} z}=\frac{1+q^{n} z+q^{2 n} z^{2}}{1-q^{3 n} z^{3}}
$$

using (2.7), we can write

$$
L\left(q^{2 / 3}\right)=\sum_{n=-\infty}^{\infty} \frac{(-1)^{n} q^{n(3 n+5) / 2}\left(q^{4 / 3}+q^{n+2}+q^{2 n+2} q^{2 / 3}\right)}{1-q^{3 n+2}}
$$

Substituting (2.11) and (2.12) into (2.10) gives

$$
\begin{aligned}
& \frac{\left(q^{6} ; q^{6}\right)_{\infty}^{2}\left(q^{3} ; q^{3}\right)_{\infty}\left[q ; q^{6}\right]_{\infty}}{(q ; q)_{\infty}} \sum_{k=0}^{2} \frac{q^{2 k / 3}\left[q^{2 k+1} ; q^{6}\right]_{\infty}}{\left[q^{2 k-1} ; q^{6}\right]_{\infty}} \\
& =\sum_{n=-\infty}^{\infty} \frac{(-1)^{n} q^{n(3 n+5) / 2}\left(q^{4 / 3}+q^{n+2}+q^{2 n+2} q^{2 / 3}\right)}{1-q^{3 n+2}} \\
& \quad-2 \psi_{-}(q)(q ; q)_{\infty}-\phi_{-}(q) q^{1 / 3}(q ; q)_{\infty}
\end{aligned}
$$


Therefore, equating the terms with integral powers of $q$ yields

$$
\begin{aligned}
2(q ; q)_{\infty} \psi_{-}(q) & =\sum_{n=-\infty}^{\infty} \frac{(-1)^{n} q^{n(3 n+7) / 2+2}}{1-q^{3 n+2}}+q \frac{\left(q^{6} ; q^{6}\right)_{\infty}^{3}}{\left(q^{2} ; q^{2}\right)_{\infty}} \\
& =\sum_{n=-\infty}^{\infty} \frac{(-1)^{n} q^{n(3 n+5) / 2+1}}{1-q^{3 n+1}}+q \frac{\left(q^{6} ; q^{6}\right)_{\infty}^{3}}{\left(q^{2} ; q^{2}\right)_{\infty}}
\end{aligned}
$$

where we replaced $n$ by $-n-1$ in the penultimate line. Similarly, equating the terms with powers of $q$ of the form $q^{n+1 / 3}$ gives

$$
(q ; q)_{\infty} \phi_{-}(q)=\sum_{n=-\infty}^{\infty} \frac{(-1)^{n} q^{n(3 n+5) / 2+1}}{1-q^{3 n+2}}-\frac{q(q ; q)_{\infty}\left(q^{6} ; q^{6}\right)_{\infty}^{6}}{\left(q^{2} ; q^{2}\right)_{\infty}^{2}\left(q^{3} ; q^{3}\right)_{\infty}^{3}}
$$

We now begin our proofs of the four identities in Theorem 1.1.

Proof of (1.7). Setting $z=q^{2}$ in (1.15) gives

$$
q\left(q^{2} ; q^{2}\right)_{\infty} \rho(q)=q \frac{\left(q^{3} ; q^{3}\right)_{\infty}^{2}}{\left(q, q^{2} ; q^{3}\right)_{\infty}}+\sum_{n=-\infty}^{\infty} \frac{(-1)^{n} q^{3 n^{2}+5 n+2}}{1-q^{6 n+2}}
$$

Therefore, by (2.13), (2.15), and the calculation (2.17) which follows below, we deduce that

$$
\begin{aligned}
\rho( & -q)+2 q^{-1} \psi_{-}\left(q^{2}\right) \\
& =\frac{1}{\left(q^{2} ; q^{2}\right)_{\infty}}\left\{\frac{\left(-q^{3} ;-q^{3}\right)_{\infty}^{2}}{\left(-q, q^{2} ;-q^{3}\right)_{\infty}}+q \frac{\left(q^{12} ; q^{12}\right)_{\infty}^{3}}{\left(q^{4} ; q^{4}\right)_{\infty}}\right\} \\
& =\frac{\left(q^{6} ; q^{12}\right)_{\infty}^{3}}{\left(q^{2} ; q^{2}\right)_{\infty}\left(q^{2} ; q^{4}\right)_{\infty}}\left\{\frac{\left(q, q^{5}, q^{6}, q^{6} ; q^{6}\right)_{\infty}}{\left(q^{2}, q^{3}, q^{3}, q^{4} ; q^{6}\right)_{\infty}}+q \frac{\left(q^{2}, q^{10}, q^{12}, q^{12} ; q^{12}\right)_{\infty}}{\left(q^{4}, q^{6}, q^{6}, q^{8} ; q^{12}\right)_{\infty}}\right\} \\
& =\frac{\left(q^{6} ; q^{12}\right)_{\infty}^{3}}{\left(q^{2} ; q^{2}\right)_{\infty}\left(q^{2} ; q^{4}\right)_{\infty}} \frac{\left(q^{12} ; q^{12}\right)_{\infty}^{2}\left(q^{4} ; q^{4}\right)_{\infty}^{2}}{\left(q^{6} ; q^{6}\right)_{\infty}\left(q^{2} ; q^{2}\right)_{\infty}} \\
& =\left(-q^{2} ; q^{2}\right)_{\infty}^{3}\left(q^{6}, q^{6}, q^{12} ; q^{12}\right)_{\infty},
\end{aligned}
$$

where in the second equality, we applied (1.12) three times to obtain

$$
\begin{aligned}
& \frac{\left(q, q^{5}, q^{6}, q^{6} ; q^{6}\right)_{\infty}}{\left(q^{2}, q^{3}, q^{3}, q^{4} ; q^{6}\right)_{\infty}}+q \frac{\left(q^{2}, q^{10}, q^{12}, q^{12} ; q^{12}\right)_{\infty}}{\left(q^{4}, q^{6}, q^{6}, q^{8} ; q^{12}\right)_{\infty}} \\
& \quad=\sum_{n=-\infty}^{\infty} \frac{q^{3 n}}{1-q^{6 n+2}}+q \sum_{n=-\infty}^{\infty} \frac{q^{6 n}}{1-q^{12 n+4}} \\
& \quad=\sum_{n=-\infty}^{\infty} \frac{q^{6 n}}{1-q^{12 n+2}}+\sum_{n=-\infty}^{\infty} \frac{q^{6 n+3}}{1-q^{12 n+8}}+q \sum_{n=-\infty}^{\infty} \frac{q^{6 n}}{1-q^{12 n+4}}
\end{aligned}
$$




$$
\begin{aligned}
& =\sum_{n=-\infty}^{\infty} \frac{q^{6 n}}{1-q^{12 n+2}}-\sum_{n=-\infty}^{\infty} \frac{q^{6 n-5}}{1-q^{12 n-8}}+q \sum_{n=-\infty}^{\infty} \frac{q^{6 n}}{1-q^{12 n+4}} \\
& =\sum_{n=-\infty}^{\infty} \frac{q^{6 n}}{1-q^{12 n+2}} \\
& =\frac{\left(q^{12} ; q^{12}\right)_{\infty}^{2}\left(q^{4} ; q^{4}\right)_{\infty}^{2}}{\left(q^{6} ; q^{6}\right)_{\infty}\left(q^{2} ; q^{2}\right)_{\infty}}
\end{aligned}
$$

Replacing $q$ by $-q$ in (2.16) then proves (1.7).

Proof of (1.8). Setting $z=q^{2}$ in (1.16), we see that

$$
\left(q^{2} ; q^{2}\right)_{\infty} \sigma(q)=q \frac{(q ; q)_{\infty}\left(q^{6} ; q^{6}\right)_{\infty}^{6}}{\left(q^{2} ; q^{2}\right)_{\infty}^{2}\left(q^{3} ; q^{3}\right)_{\infty}^{3}}+\sum_{n=-\infty}^{\infty} \frac{(-1)^{n} q^{n(3 n+5)+2}}{1-q^{6 n+4}}
$$

Therefore, by (2.18) and (2.14),

$$
\left(q^{2} ; q^{2}\right)_{\infty} \sigma(q)-\left(q^{2} ; q^{2}\right)_{\infty} \phi_{-}\left(q^{2}\right)=q \frac{\left(q, q^{5}, q^{6}, q^{6} ; q^{6}\right)_{\infty}}{\left(q^{2}, q^{3}, q^{3}, q^{4} ; q^{6}\right)_{\infty}}+q^{2} \frac{\left(q^{2}, q^{10}, q^{12}, q^{12} ; q^{12}\right)_{\infty}}{\left(q^{4}, q^{6}, q^{6}, q^{8} ; q^{12}\right)_{\infty}}
$$

which, after invoking (2.17) and dividing by $\left(q^{2} ; q^{2}\right)_{\infty}$, gives the equivalent identity

$$
\sigma(q)-\phi_{-}\left(q^{2}\right)=q \frac{\left(q^{12} ; q^{12}\right)_{\infty}^{2}\left(q^{4} ; q^{4}\right)_{\infty}^{2}}{\left(q^{6} ; q^{6}\right)_{\infty}\left(q^{2} ; q^{2}\right)_{\infty}^{2}}=q\left(-q^{2} ; q^{2}\right)_{\infty}^{2}\left(-q^{6},-q^{6}, q^{6} ; q^{6}\right)_{\infty}
$$

Thus, (1.8) has been established.

Proof of (1.9). Substitute $z=q^{2}$ in (1.18) and use the Jacobi triple product identity (1.11) to deduce that

$$
\begin{aligned}
2\left(q^{2} ; q^{2}\right)_{\infty} \mu(q)= & \frac{2\left(q^{6} ; q^{6}\right)_{\infty}^{3}\left(q^{6} ; q^{12}\right)_{\infty}\left(-q^{2},-q^{4} ; q^{6}\right)_{\infty}^{2}}{\left(q^{4} ; q^{4}\right)_{\infty}}-\frac{f(-q,-q) f\left(q, q^{2}\right)\left(q^{2} ; q^{2}\right)_{\infty}}{f\left(q, q^{3}\right)} \\
& -4 \sum_{n=-\infty}^{\infty} \frac{(-1)^{n} q^{n(3 n+5)+2}}{1-q^{6 n+4}} \\
= & \frac{2\left(q^{4} ; q^{4}\right)_{\infty}\left(q^{6} ; q^{6}\right)_{\infty}^{6}}{\left(q^{2} ; q^{2}\right)_{\infty}^{2}\left(q^{12} ; q^{12}\right)_{\infty}^{3}}-\frac{(q ; q)_{\infty}^{2}\left(q^{3} ; q^{3}\right)_{\infty}^{2}}{\left(q^{2} ; q^{2}\right)_{\infty}\left(q^{6} ; q^{6}\right)_{\infty}} \\
& -4 \sum_{n=-\infty}^{\infty} \frac{(-1)^{n} q^{n(3 n+5)+2}}{1-q^{6 n+4}},
\end{aligned}
$$

while replacing $q$ by $q^{2}$ in (2.14) gives

$$
\left(q^{2} ; q^{2}\right)_{\infty} \phi_{-}\left(q^{2}\right)=\sum_{n=-\infty}^{\infty} \frac{(-1)^{n} q^{n(3 n+5)+2}}{1-q^{6 n+4}}-\frac{q^{2}\left(q^{2} ; q^{2}\right)_{\infty}\left(q^{12} ; q^{12}\right)_{\infty}^{6}}{\left(q^{4} ; q^{4}\right)_{\infty}^{2}\left(q^{6} ; q^{6}\right)_{\infty}^{3}}
$$


Therefore, with three applications of (1.12),

$$
\begin{aligned}
& 4\left(q^{2} ; q^{2}\right)_{\infty} \phi_{-}\left(q^{2}\right)+2\left(q^{2} ; q^{2}\right)_{\infty} \mu(q) \\
& =2 \frac{\left(q^{4} ; q^{4}\right)_{\infty}\left(q^{6} ; q^{6}\right)_{\infty}^{6}}{\left(q^{2} ; q^{2}\right)_{\infty}^{2}\left(q^{12} ; q^{12}\right)_{\infty}^{3}}-\frac{(q ; q)_{\infty}^{2}\left(q^{3} ; q^{3}\right)_{\infty}^{2}}{\left(q^{2} ; q^{2}\right)_{\infty}\left(q^{6} ; q^{6}\right)_{\infty}} \\
& -4 \frac{q^{2}\left(q^{2} ; q^{2}\right)_{\infty}\left(q^{12} ; q^{12}\right)_{\infty}^{6}}{\left(q^{4} ; q^{4}\right)_{\infty}^{2}\left(q^{6} ; q^{6}\right)_{\infty}^{3}} \\
& =4 \frac{\left(-q^{2},-q^{4}, q^{6}, q^{6} ; q^{6}\right)_{\infty}}{\left(q^{2}, q^{4},-1,-q^{6} ; q^{6}\right)_{\infty}}-2 \frac{\left(q, q^{2}, q^{3}, q^{3} ; q^{3}\right)_{\infty}}{\left(-q,-q^{2},-1,-q^{3} ; q^{3}\right)_{\infty}} \\
& -4 \frac{q^{2}\left(q^{2}, q^{10}, q^{12}, q^{12} ; q^{12}\right)_{\infty}}{\left(q^{4}, q^{8}, q^{6}, q^{6} ; q^{12}\right)_{\infty}} \\
& =4 \sum_{n=-\infty}^{\infty} \frac{q^{2 n}}{1+q^{6 n}}-2 \sum_{n=-\infty}^{\infty} \frac{(-q)^{n}}{1+q^{3 n}}-4 \sum_{n=-\infty}^{\infty} \frac{q^{4 n+2}}{1-q^{12 n+6}} \\
& =4 \sum_{n=-\infty}^{\infty} \frac{q^{2 n}}{1+q^{6 n}}-2 \sum_{n=-\infty}^{\infty} \frac{q^{2 n}}{1+q^{6 n}}+2 \sum_{n=-\infty}^{\infty} \frac{q^{2 n+1}}{1+q^{6 n+3}}+4 \sum_{n=-\infty}^{\infty} \frac{q^{8 n+4}}{1-q^{12 n+6}} \\
& =2 \sum_{n=-\infty}^{\infty} \frac{q^{2 n}}{1+q^{6 n}}+2 \sum_{n=-\infty}^{\infty} \frac{q^{2 n+1}\left(1-q^{6 n+3}\right)}{1-q^{12 n+6}}+4 \sum_{n=-\infty}^{\infty} \frac{q^{8 n+4}}{1-q^{12 n+6}} \\
& =2 \sum_{n=-\infty}^{\infty} \frac{q^{2 n}}{1+q^{6 n}}+2 \sum_{n=-\infty}^{\infty} \frac{q^{2 n+1}}{1-q^{6 n+3}}=2 \sum_{n=-\infty}^{\infty} \frac{q^{n}}{1+\left(-q^{3}\right)^{n}} \\
& =2 \frac{\left(-q, q^{2},-q^{3},-q^{3} ;-q^{3}\right)_{\infty}}{\left(q,-q^{2},-1, q^{3} ;-q^{3}\right)_{\infty}}=\left(-q,-q, q^{2} ; q^{2}\right)_{\infty}\left(-q^{3},-q^{3}, q^{6} ; q^{6}\right)_{\infty}
\end{aligned}
$$

where, in the penultimate equality, we applied (1.12) again. Therefore, dividing throughout the last series of equalities by $\left(q^{2} ; q^{2}\right)_{\infty}$, we complete the proof.

Proof of (1.10). Substituting $z=q^{2}$ in (1.17) gives

$$
\begin{aligned}
q\left(q^{2} ; q^{2}\right)_{\infty} \lambda(q)= & \frac{2 q^{2}\left(q^{12} ; q^{12}\right)_{\infty}^{2}\left(q^{2} ; q^{4}\right)_{\infty}^{2}\left[-q^{4},-1 ; q^{6}\right]_{\infty}}{\left[q^{2} ; q^{6}\right]_{\infty}} \\
& +\frac{q(q ; q)_{\infty}\left(q^{6} ; q^{6}\right)_{\infty}^{2}\left[q, q^{2} ; q^{6}\right]_{\infty}}{\left(-q,-q^{3}, q^{4} ; q^{4}\right)_{\infty}}-2 \sum_{n=-\infty}^{\infty} \frac{(-1)^{n} q^{n(3 n+5)+2}}{1-q^{6 n+2}}
\end{aligned}
$$

while replacing $q$ by $q^{2}$ in (2.13) yields

$$
2\left(q^{2} ; q^{2}\right)_{\infty} \psi_{-}\left(q^{2}\right)=\sum_{n=-\infty}^{\infty} \frac{(-1)^{n} q^{n(3 n+5)+2}}{1-q^{6 n+2}}+q^{2} \frac{\left(q^{12} ; q^{12}\right)_{\infty}^{3}}{\left(q^{4} ; q^{4}\right)_{\infty}}
$$

Thus, applying (1.12) in the fourth, seventh, ninth, and penultimate equalities below, we find that 


$$
\begin{aligned}
& 4\left(q^{2} ; q^{2}\right)_{\infty} \psi_{-}\left(q^{2}\right)+q\left(q^{2} ; q^{2}\right)_{\infty} \lambda(q) \\
& =\frac{4 q^{2}\left(q^{12} ; q^{12}\right)_{\infty}^{2}}{\left(q^{4}, q^{8} ; q^{12}\right)_{\infty}}+\frac{q\left(q ; q^{2}\right)_{\infty}\left(q^{6} ; q^{6}\right)_{\infty}(q ; q)_{\infty}^{2}}{\left(q^{3} ; q^{6}\right)_{\infty}\left(q^{2}, q^{2}\right)_{\infty}}+\frac{2 q^{2}\left(q^{12} ; q^{12}\right)_{\infty}^{2}}{\left(q^{4}, q^{8} ; q^{12}\right)_{\infty}} \\
& =\frac{6 q^{2}\left(q^{12} ; q^{12}\right)_{\infty}^{2}}{\left(q^{4}, q^{8} ; q^{12}\right)_{\infty}}+\frac{q\left(q ; q^{2}\right)_{\infty}\left(q^{6} ; q^{6}\right)_{\infty}(q ; q)_{\infty}^{2}}{\left(q^{3} ; q^{6}\right)_{\infty}\left(q^{2}, q^{2}\right)_{\infty}} \\
& =\frac{3 q^{2}\left(-1,-q^{6}, q^{6}, q^{6} ; q^{6}\right)_{\infty}}{\left(q^{2}, q^{4},-q^{2},-q^{4} ; q^{6}\right)_{\infty}}+\frac{q\left(q, q^{2}, q^{3}, q^{3} ; q^{3}\right)_{\infty}}{\left(-q,-q^{2},-q,-q^{2} ; q^{3}\right)_{\infty}} \\
& =3 \sum_{n=-\infty}^{\infty} \frac{q^{2 n+2}}{1+q^{6 n+4}}+q \sum_{n=-\infty}^{\infty} \frac{(-q)^{n}}{1+q^{3 n+1}} \\
& =3 \sum_{n=-\infty}^{\infty} \frac{q^{2 n+2}}{1+q^{6 n+4}}+\sum_{n=-\infty}^{\infty} \frac{q^{2 n+1}}{1+q^{6 n+1}}-\sum_{n=-\infty}^{\infty} \frac{q^{2 n+2}}{1+q^{6 n+4}} \\
& =\sum_{n=-\infty}^{\infty} \frac{q^{2 n+2}}{1+q^{6 n+4}}+\sum_{n=-\infty}^{\infty} \frac{q^{n+1}}{1+q^{3 n+1}} \\
& =q^{2} \frac{\left(-1,-q^{6}, q^{6}, q^{6} ; q^{6}\right)_{\infty}}{\left(q^{2}, q^{4},-q^{2},-q^{4} ; q^{6}\right)_{\infty}}+q \frac{\left(-q,-q^{2}, q^{3}, q^{3} ; q^{3}\right)_{\infty}}{\left(q, q^{2},-q,-q^{2} ; q^{3}\right)_{\infty}} \\
& =q^{2} \frac{\left(-1,-q^{6}, q^{6}, q^{6} ; q^{6}\right)_{\infty}}{\left(q^{2}, q^{4},-q^{2},-q^{4} ; q^{6}\right)_{\infty}}+q \frac{\left(q^{3}, q^{3}, q^{6}, q^{6} ; q^{6}\right)_{\infty}}{\left(q, q^{5}, q^{2}, q^{4} ; q^{6}\right)_{\infty}} \\
& =\sum_{n=-\infty}^{\infty} \frac{(-1)^{n} q^{4 n+2}}{1-q^{6 n+2}}+\sum_{n=-\infty}^{\infty} \frac{q^{n+1}}{1-q^{6 n+2}} \\
& =\sum_{n=-\infty}^{\infty} \frac{q^{8 n+2}}{1-q^{12 n+2}}-\sum_{n=-\infty}^{\infty} \frac{q^{8 n+6}}{1-q^{12 n+8}}+\sum_{n=-\infty}^{\infty} \frac{q^{2 n+1}}{1-q^{12 n+2}}+\sum_{n=-\infty}^{\infty} \frac{q^{2 n+2}}{1-q^{12 n+8}} \\
& =\sum_{n=-\infty}^{\infty} \frac{q^{2 n+1}\left(1+q^{6 n+1}\right)}{1-q^{12 n+2}}+\sum_{n=-\infty}^{\infty} \frac{q^{2 n+2}\left(1-q^{6 n+4}\right)}{1-q^{12 n+8}} \\
& =\sum_{n=-\infty}^{\infty} \frac{q^{2 n+1}}{1-q^{6 n+1}}+\sum_{n=-\infty}^{\infty} \frac{q^{2 n+2}}{1+q^{6 n+4}} \\
& =\sum_{n=-\infty}^{\infty} \frac{q^{n+1}}{1-(-1)^{n} q^{3 n+1}}=q \frac{\left(q^{2},-q,-q^{3},-q^{3} ;-q^{3}\right)_{\infty}}{\left(q,-q^{2}, q,-q^{2} ;-q^{3}\right)_{\infty}} \\
& =q\left(q^{2} ; q^{2}\right)_{\infty}\left(-q ; q^{2}\right)_{\infty}^{3}\left(q^{3}, q^{9}, q^{12} ; q^{12}\right)_{\infty} \text {. }
\end{aligned}
$$

We close this section by proving the two analogs of (1.18) that we mentioned at the end of the Introduction.

From [9, Eq. (4.4)], with $q$ replaced by $q^{3}$, we find that, after rearranging, 


$$
\begin{aligned}
\frac{\left[a, c / b ; q^{3}\right]_{\infty}\left(q^{3} ; q^{3}\right)_{\infty}^{2}}{\left[b, c, a / b ; q^{3}\right]_{\infty}}= & \sum_{n=-\infty}^{\infty} \frac{(-1)^{n} q^{3 n(n+1) / 2} a^{n} c^{-n}}{1-b q^{3 n}} \\
& -\frac{c}{b} \frac{\left[a / c ; q^{3}\right]_{\infty}}{\left[a / b ; q^{3}\right]_{\infty}} \sum_{n=-\infty}^{\infty} \frac{(-1)^{n} q^{3 n(n+1) / 2} a^{n} b^{-n}}{1-c q^{3 n}}
\end{aligned}
$$

Setting $a=c q$ and $b=q^{2}$ in (2.19), we find that

$$
\begin{aligned}
\frac{\left[c q, c / q^{2} ; q^{3}\right]_{\infty}\left(q^{3} ; q^{3}\right)_{\infty}^{2}}{\left[q, c, c / q ; q^{3}\right]_{\infty}}= & \sum_{n=-\infty}^{\infty} \frac{(-1)^{n} q^{(3 n+5) / 2}}{1-q^{3 n+2}} \\
& -\frac{c}{q^{2}} \frac{\left[q ; q^{3}\right]_{\infty}}{\left[c / q ; q^{3}\right]_{\infty}} \sum_{n=-\infty}^{\infty} \frac{(-1)^{n} q^{n(3 n+1) / 2} c^{n}}{1-c q^{3 n}}
\end{aligned}
$$

Thus, substituting the first series on the right side with (2.14), we find that

$$
\begin{aligned}
q \frac{\left[c q, c / q^{2} ; q^{3}\right]_{\infty}\left(q^{3} ; q^{3}\right)_{\infty}^{2}}{\left[q, c, c / q ; q^{3}\right]_{\infty}}= & (q ; q)_{\infty} \phi_{-}(q)+\frac{q(q ; q)_{\infty}\left(q^{6} ; q^{6}\right)_{\infty}^{6}}{\left(q^{2} ; q^{2}\right)_{\infty}^{2}\left(q^{3} ; q^{3}\right)_{\infty}^{3}} \\
& -\frac{c}{q} \frac{\left[q ; q^{3}\right]_{\infty}}{\left[c / q ; q^{3}\right]_{\infty}} \sum_{n=-\infty}^{\infty} \frac{(-1)^{n} q^{n(3 n+1) / 2} c^{n}}{1-c q^{3 n}}
\end{aligned}
$$

After replacing $c$ by $c q$ and rearranging, we see that

$$
\begin{aligned}
\left(c, q^{3} / c, q^{3} ; q^{3}\right)_{\infty} \phi_{-}(q)= & q \frac{\left[c q^{2}, c / q ; q^{3}\right]_{\infty}\left(q^{3} ; q^{3}\right)_{\infty}^{2}}{\left[q, q, c q ; q^{3}\right]_{\infty}}-q \frac{\left(q^{6} ; q^{6}\right)_{\infty}^{6}\left[c ; q^{3}\right]_{\infty}}{\left(q^{2} ; q^{2}\right)_{\infty}^{2}\left(q^{3} ; q^{3}\right)_{\infty}^{2}} \\
& +\sum_{n=-\infty}^{\infty} \frac{(-1)^{n} q^{3 n(n+1) / 2} c^{n+1}}{1-c q^{3 n+1}}
\end{aligned}
$$

Similarly, setting $a=c q$ and $b=q$ in (2.19), we find that

$$
\frac{\left[c q, c / q ; q^{3}\right]_{\infty}\left(q^{3} ; q^{3}\right)_{\infty}^{2}}{\left[q, c, c ; q^{3}\right]_{\infty}}=\sum_{n=-\infty}^{\infty} \frac{(-1)^{n} q^{n(3 n+5) / 2}}{1-q^{3 n+1}}-\frac{c}{q} \frac{\left[q ; q^{3}\right]_{\infty}}{\left[c ; q^{3}\right]_{\infty}} \sum_{n=-\infty}^{\infty} \frac{(-1)^{n} q^{3 n(n+1) / 2} c^{n}}{1-c q^{3 n}}
$$

which, upon substituting the first series on the right side with (2.13) and rearranging, yields

$$
\begin{aligned}
2\left(c, q^{3} / c, q^{3} ; q^{3}\right)_{\infty} \psi_{-}(q)= & q \frac{\left(c, q^{3} / c, q^{3} ; q^{3}\right)_{\infty}\left(q^{6} ; q^{6}\right)_{\infty}^{3}}{(q ; q)_{\infty}\left(q^{2} ; q^{2}\right)_{\infty}}+q \frac{\left[c q, c / q ; q^{3}\right]_{\infty}\left(q^{3} ; q^{3}\right)_{\infty}^{2}}{\left[q, q, c ; q^{3}\right]_{\infty}} \\
& +\sum_{n=-\infty}^{\infty} \frac{(-1)^{n} q^{3 n(n+1) / 2} c^{n+1}}{1-c q^{3 n}}
\end{aligned}
$$




\section{An identity on page 4 of Ramanujan's lost notebook}

The last entry on page 4 of Ramanujan's lost notebook is given by

$$
\sum_{n=0}^{\infty} \frac{(-1)^{n}\left(q^{3} ; q^{6}\right)_{n}}{\left(-q^{3} ; q^{3}\right)_{n}^{2}}-2 \psi(q)=\frac{\varphi^{2}(-q)}{2 \Psi\left(q^{3}\right)}
$$

where

$$
\varphi(q):=\sum_{n=-\infty}^{\infty} q^{n^{2}} \quad \text { and } \quad \Psi(q):=\sum_{n=0}^{\infty} q^{n(n+1) / 2}=\frac{\left(q^{2} ; q^{2}\right)_{\infty}}{\left(q ; q^{2}\right)_{\infty}}
$$

where the product representation, which we use several times without a comment in our derivations below, follows from (1.11); see also [4, p. 36, Entry 22(iii)]. Since the series on the left-hand side of (3.1) does not converge, at present, Ramanujan's claim is meaningless. We provide a meaningful interpretation of this hitherto unexamined entry.

Following [2, p. 266, Entry 12.3.3], we use the identity [2, Eq. (12.3.6)]

$$
\begin{aligned}
& \lim _{\alpha \rightarrow 1^{-}} \sum_{n=0}^{\infty} \frac{(-1)^{n}(\alpha q ; q)_{n}\left(q ; q^{2}\right)_{n}}{(q ; q)_{n}(-\alpha a q ; q)_{n}(-\alpha q / a ; q)_{n}} \alpha^{n} \\
& \quad=\frac{\left(q ; q^{2}\right)_{\infty}}{2\left(q^{2} ; q^{2}\right)_{\infty}} \sum_{n=-\infty}^{\infty} \frac{(1+1 / a)(1+a) q^{n(n+1) / 2}}{\left(1+a q^{n}\right)\left(1+q^{n} / a\right)}
\end{aligned}
$$

which, in fact, is an interpretation of another identity in [13, p. 4], which formerly was without meaning. Putting $a=1$ and replacing $q$ by $q^{3}$ in (3.2), we find that the left-hand side of (3.1) should likely be interpreted as

$$
\begin{aligned}
\sum_{n=0}^{\infty} \frac{(-1)^{n}\left(q^{3} ; q^{6}\right)_{n}}{\left(-q^{3} ; q^{3}\right)_{n}^{2}} & =\lim _{\alpha \rightarrow 1^{-}} \sum_{n=0}^{\infty} \frac{(-1)^{n}\left(\alpha q^{3} ; q^{3}\right)_{n}\left(q^{3} ; q^{6}\right)_{n}}{\left(q^{3} ; q^{3}\right)_{n}\left(-\alpha q^{3} ; q^{3}\right)_{n}^{2}} \alpha^{n} \\
& =\frac{2}{\Psi\left(q^{3}\right)} \sum_{n=-\infty}^{\infty} \frac{q^{3 n(n+1) / 2}}{\left(1+q^{3 n}\right)^{2}} \\
& =\frac{2}{\Psi\left(q^{3}\right)}\left\{\frac{1}{4}+\sum_{n=1}^{\infty} \frac{q^{3 n(n+1) / 2}}{1+q^{3 n}}\right\} \\
& =\frac{1}{\Psi\left(q^{3}\right)} \sum_{n=-\infty}^{\infty} \frac{q^{3 n(n+1) / 2}}{1+q^{3 n}},
\end{aligned}
$$

thus enabling (3.1) to be expressed in the following equivalent form.

$$
\sum_{n=-\infty}^{\infty} \frac{q^{3 n(n+1) / 2}}{1+q^{3 n}}-2 \Psi\left(q^{3}\right) \psi(q)=\frac{1}{2} \varphi^{2}(-q)
$$


Setting $z=-1$ in (1.14), we obtain an equivalent form of (3.4), and this completes the proof of a corrected version of (3.1).

As in [10], by using (3.3), identities similar to (3.1) can be easily obtained. However, they probably are not found in Ramanujan's lost notebook. For example, by substituting $z=-1$ in both (1.15) and (1.17), applying (3.3) with $q$ replaced by $q^{2}$, dividing by $\Psi\left(q^{6}\right)$, and rearranging, we obtain, respectively,

$$
\sum_{n=0}^{\infty} \frac{(-1)^{n}\left(q^{6} ; q^{12}\right)_{n}}{\left(-q^{6} ; q^{6}\right)_{n}^{2}}+2 q \rho(q)=\frac{\varphi^{2}\left(-q^{3}\right) \varphi^{2}\left(-q^{2}\right)}{2 \Psi\left(q^{6}\right) \varphi^{2}(-q)}
$$

and

$$
\sum_{n=0}^{\infty} \frac{(-1)^{n}\left(q^{6} ; q^{12}\right)_{n}}{\left(-q^{6} ; q^{6}\right)_{n}^{2}}-q \lambda(q)=\frac{\varphi^{2}\left(-q^{2}\right)}{2 \Psi\left(q^{6}\right)}-\frac{q \Psi\left(q^{3}\right) \varphi(-q)}{\Psi(q)}
$$

\section{Acknowledgment}

We are grateful to Kathrin Bringmann for pointing out an error in our original formulation of (1.8).

\section{References}

[1] G.E. Andrews, On basic hypergeometric series, mock theta functions and partitions (I), Q. J. Math. 17 (1966) 64-80.

[2] G.E. Andrews, B.C. Berndt, Ramanujan’s Lost Notebook, Part I, Springer, New York, 2005.

[3] G.E. Andrews, D. Hickerson, Ramanujan's 'lost' notebook VII: The sixth order mock theta functions, Adv. Math. 89 (1991) 60-105.

[4] B.C. Berndt, Ramanujan's Notebooks, Part III, Springer-Verlag, New York, 1991.

[5] B.C. Berndt, R.A. Rankin, Ramanujan: Letters and Commentary, American Mathematical Society, Providence, RI, 1995, London Mathematical Society, London, 1995.

[6] K. Bringmann, K. Ono, The $f(q)$ mock theta function conjecture and partition ranks, Invent. Math. 165 (2006) 243-266.

[7] K. Bringmann, K. Ono, Lifting elliptic cusp forms to Maass forms with an application to partitions, Proc. Natl. Acad. Sci. USA 104 (2007) 3725-3731.

[8] K. Bringmann, K. Ono, Dyson's ranks and Maass forms, Ann. of Math., in press.

[9] S.H. Chan, Generalized Lambert series identities, Proc. London Math. Soc. (3) 91 (2005) 598-622.

[10] Y.-S. Choi, Identities for Ramanujan's sixth-order mock theta functions, Q. J. Math. 53 (2002) 147-159.

[11] R.J. McIntosh, Modular transformations of Ramanujan's sixth order mock theta functions, preprint.

[12] S. Ramanujan, Collected Papers, Cambridge University Press, Cambridge, 1927; reprinted by Chelsea, New York, 1962; reprinted by the American Mathematical Society, Providence, RI, 2000.

[13] S. Ramanujan, The Lost Notebook and Other Unpublished Papers, Narosa, New Delhi, 1988.

[14] G.N. Watson, The final problem: An account of the mock theta functions, J. London Math. Soc. 11 (1936) 55-80; reprinted in: B.C. Berndt, R.A. Rankin, Ramanujan: Essays and Surveys, American Mathematical Society, Providence, 2001, London Mathematical Society, London, 2001. 